\title{
Joint Data Link System Based on Cloud Computing
}

\author{
Yang Juan \\ The 3rd Department, PLA.Chongqing Communication \\ College, Chongqing, China \\ College of Communication Engineering, Chongqing \\ University, Chongqing, China \\ Email: yajj6688@yahoo.com.cn
}

\author{
Xinyu Chen and Bing Jiang and Jianlu Luo and \\ Wenjing Zhang \\ The 3rd Department, PLA.Chongqing Communication \\ College, Chongqing, China \\ The 3rd Department, PLA.Chongqing Communication \\ College, Chongqing, China \\ The Dept. of Electronic Technology, PAP Police College, \\ Chengdu, China \\ The 3rd Department, PLA.Chongqing Communication \\ College, Chongqing, China \\ Email: $\{$ chenxiny1800@126.com, \\ jb_yxx@163.com,lojel@tccxfw.com ,602382254@qq.com
}

\begin{abstract}
In order to meet the requirements of multiple data links cooperative combat in network centric battle field, we analyzed the problems and challenges that US Army tactical data link system encountered when meeting the needs of network-centric warfare, and elaborated the turning point that the ideas of cloud computing service mode construct the networking data link system in network-centric battle field. On the basis of this, we put forward a new battlefield-oriented, demand-based, real-time serviced networking data link system---the joint data link system based on cloud computing, and proved that the use of cloud technology in Joined Data Links can improve the system service level markedly.
\end{abstract}

Keywords-network centric warfare, cloud computing, joint data link system

\section{INTRODUCTION}

In network centric warfare, tactical data links such as link-4A 、 link-10、 link-11 、 link-16 and link-22 can no longer meet the requirement of real time high quality network data transmission and sharing between multi-nodes. Therefore, US army planned to build complete networking data links----Joined Data Links in 2015 to implement multilinks military cooperation and to provide real-time and complete battlefield information to end users ${ }^{[1]}$. In Joined Data Links, US army strengthens battlefield weaponry cooperation and information fusion across different links, to provide precise and real-time battlefield information satisfying fire control requirement, and to implement cooperative campaign with different weapon systems. It puts different closed-loop data links into one integration, in which the data links can inter-connect and inter-operate with each other, and provides high quality and real-time battlefield information for network-centric warfare ${ }^{[2]}$ on demand

The first important thing to build Joined Data Links is to implement inter-connection, inter-communication and interoperation. However, most data links in US army are designed to accomplish their own missions, and have different information type, data precision, employ scope, transmission distance, anti-jamming ability and etc. with each other., forming a mission oriented "chimney" system.
At the moment US army mainly has three methods to achieve inter-connection, inter-communication and interoperation between these "chimney" data links ${ }^{[3,4]}$ :point to point conversion, requiring different system terminals have the same common data base; common data base or common information type notation, requiring common information format for different data links, very poor in feasibility and maneuverability; gateways, requiring either to modify each terminal, or to apply a "chimney" gateway, very poor in generality and expandability, and being easy to become the communication bottleneck due to its vulnerability to attacks. Apparently, none of the above methods can provide cooperative campaign ability across battlefield platforms, not to mention transferring "right information" in "right format" to "right receiver" at "right time" and suppressing the same attempt from the enemy, which transforms information acquiring ability to scientific decision making ability and warfare ability to the most extend proposed by network centric warfare theory.

Furthermore, according to network centric warfare theory, Joined Data Links is facing challenges more than that ${ }^{[5-7]}$ :Network-centric warfare requires Joined Data Links to distribute battlefield information as desired, highly real-time information exchange ${ }^{[8-10]}$, seamless connection between sub-links and expandability of the whole Joined Data Links ${ }^{[11]}$, and Joined Data Links to have strong survivability.

However, researches all over the world till now do not satisfy all the requirements above.

Cloud computing, a new computing mode, provides dynamically flexible virtualized resource service to users by internet $^{[12},{ }^{13]}$.Joined Data Links based on cloud computing has not been seen in any report. The main idea to build Joined Data Links using cloud computing is to design a transparent and open network architecture to friendly troops. In this architecture, on one hand, distributive processing between and within sub-links is fully utilized to improve battle efficiency and network survivability, and upgrading on current software and hardware is compatible with original tactical data links and all other communication systems; on the other hand, 
defining standard interface insures that the function of current equipment will not be affected in expansion of Joined Data Links. Thus network-centric warfare battlefield multi-link cooperation is provided with seamless, survival, expandable and reliable distant information transmission network, hence its demands being satisfied.

\section{FEASIBILITY STUDY ON BUILDING JOINED DATA LINKS BASED ON CLOUD COMPUTING}

Joined Data Links has features of large scale, difference in sub-link structure, high complexity, and large scale of distribution. Now we will discuss feasibility of building Joined Data Links based on cloud computing, taking key technologies of cloud computing into account.

\section{A. Massive resources with different structures can be} optimized and integrated into Joined Data Links

Currently most tactical data link systems are based on requirements of local battle departments. Existence of different platforms, application systems and data formats makes battlefield information separated and varied in structure, vertical communication hard and horizontal sharing impossible. Cloud computing does not require computing equipment to have much commonness in software or hardware, most of which can be integrated into computing platform [14]. Therefore, it is possible to use cloud computing to thoroughly integrate different types of tactical data links, distributed across large areas and belonging to different organizations, into Joined Data Links.

\section{B. Infrastructure of Joined Data Links can be managed automatically}

The data center of current tactical data links is centralized, usually based on computer cluster in command post, and bears great risk of being destroyed by enemies and inevitability of accident hardware damage; in addition, infrastructure of Joined Data Links is of large scale and amount, distributed in different areas, simultaneously running multiple applications. It is a big challenge to effectively manage this infrastructure and decrease running cost of data center in battlefield. Cloud computing platform management technology makes large amount of servers in Joined Data Links work cooperatively. And this makes easy deployment, rapid system failure detection and repairmen possible. It also realize reliable management of large scale Joined Data Links through robotized and intellectualized methods ${ }^{[15]}$.

\section{Massive battlefield data can be stored reliably in distribution}

Information transmitted in Joined Data Links and acquired from battlefield data acquisition systems and monitoring systems, is of many types and very large amount. However data storage and management of current tactical data links cannot meet the requirement of storage and management of massive battlefield data. Cloud computing takes massive data into distributive storage, and ensures data reliability by redundant storage and highly reliable software. In cloud computing, widely used data storage nodes fall in three categories ${ }^{[16,17]}$, Therefore, cloud computing meets requirement of massive battlefield data reliable storage from Joined Data Links.

\section{Battlefield information of multi data links can be managed effectively}

Battlefield information of Joined Data Links is distributed in large areas and of large amount of types, including massive real time data such as battlefield situation, deployment, geological information, meteorological information, intelligence information, service information, command, control and cooperation, which makes requirement of processing performance of real time data much higher than that of off line data. Cloud computing data management technology satisfies distributive and varies data real-time processing and analyzing requirement. For example: BigTable ${ }^{[18]}$ from Google is designed to meet varies massive service requests which satisfies the requirement of Joined Data Links discussed above. Therefore, cloud computing effectively manages varies data with different quality requires in Joined Data Links.

\section{E. Distributive computing and analyzing of Joined Data Links can be done in high speed}

To ensure Joined Data Links run reliably, it is necessary to implement large amount of computing, analyzing, planning, optimizing, designing, decision making and mission deployment on base of massive data provided by Joined Data Links. As a new parallel programming model applied on cloud computing platform, Bigtable is capable of parallel computing a large amount of data collection. Bigtable is a sparse, distributive, longtermed, multi-dimensional, and sorting Map, satisfying requirements of batch processing, real time response, high speed data return and etc. Therefore, Bigtable is able to finish parallel computing and analyzing of Joined Data Links in high speed.

\section{F. Expandability and survivability of Joined Data Links can be improved}

Cloud computing achieves two important goals of distributive computing: expandability and high robustness $[19,20]$. Expandability means cloud computing can be seamlessly expanded to run on a large cluster, which may include thousands of nodes. Moreover, when expanding, only indispensable equipment need to be added, which greatly saves expense on hardware purchasing. High robustness means tolerance of node fault, even a large group of invalid nodes not affecting system running correctly, which meet the need of network-centric warfare.

In summary, building Joined Data Links on basis of cloud computing architecture is feasible, and is able to effectively solve problems of resource integration, distributive storage of massive data and high speed of parallel computing in network-centric warfare, thus solves the key problems of inter-connection, inter-working, interoperation and efficient real time battlefield information sharing as desired. 


\section{ARCHITECTURE OF JOINED DATA LINKS BASED ON CLOUD COMPUTING}

We will build architecture of Joined Data Links based on cloud computing, and the cross-link interacting cloud service layer in the architecture will be discussed.

\section{A. Architecture of JDLCC(Joined Data Links based on Cloud Computing )}

First, we insist that Joined Data Links is the kernel communication system in network-centric warfare. And then, according to current tactical data links' features and technology used, making reference to TCP/IP model, we add Network Layer, Cross-link Interacting Cloud Service Layer and Credibility Control Dimension(Figure 1) into data link's three-layer architecture ${ }^{[3]}$. The new architecture is networked, and it's Cross-link Interacting Cloud Service Layer provides unified technology standards. It makes sub link's computing function running in network, makes battlefield application and service intellectualized, in order to achieve the goal of "omnipresent and omnipotent" cooperation and intellectual campaign. Our design is a 5level architecture, from bottom to top: Physical Layer, Link Layer, Network Layer, Cross-link Interacting Cloud Service Layer and Application layer.

\section{B. Definition of Layers (Dimensions) in JDLSCC}

According to Figure 1, definition of JDLSCC layers is shown below:

\section{1) Application Layer}

It includes a specific collection of applications that must be needed to accomplish some specific battlefield tasks, such as aerial defense applications, missile defense applications, long-distance precise strike applications and other tactical and strategic battle applications. It also includes some common and specific applications utilizing tactical data for specific missions, such as monitoring, PPLI, mission management, weapons cooperation, encrypted audio and etc.

The functions of Application Layer are:

a) Convert data from Cross-link Interacting Cloud Service Layer into information for network campaign stuff or weaponry platform;

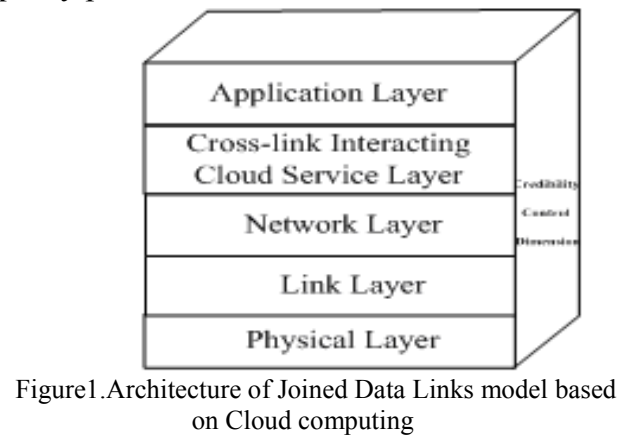

b) Break down battle into several specific steps and determine applications needed to finish these steps;

c) Inform Cross-link Interacting Cloud Service Layer which applications to call.

\section{2) Cross-link Interacting Cloud Service Layer}

Comparing to traditional tactical data links, new service is present, "cloud computing", so network of Joined Data Links now has powerful computing and information sharing capability. In this layer, the idea of cloud computing is employed to organize and utilize physical infrastructure, implementing control and management of virtual resources; by IaaS, PaaS and SaaS, services for Application Layer are provided. It mainly consists of three steps: first, encapsulate virtual battlefield resources into cloud services and publish them; then, choose deployment mode for each of different types of cloud services, and apply intellectual and efficient management to the cloud services, such as intellectual matching, dynamic assembling, fault tolerance management and etc.; last, provide battlefield users with the services when needed, such as maneuver administration and alteration management, shown in Figure 2.

In Cross-link Interacting Cloud Service Layer, resources and strategies can be processed centrally, and varied protocols are supported in order to implement universality and intellectual control. Computing capability can be got at any place in battlefield to meet the request of information acquisition and computing as desired at any time and any place, and the procedure is completely unilateral and transparent to battlefield users. Cross-link Interacting Cloud Service Layer focuses on building battlefield user oriented computing environment, so as to integrate Joined Data Links into all segments of network-centric warfare, and to provide all-dimensional and stereo service for battlefield users.

\section{3) Network Layer}

According to characteristics of sub-links, through routing computing, building reliable cross link service of data processing, transmitting and rendering, can ensure that every piece of battlefield information arrives at the right destination and implements unilateral transparent transmission of battlefield information. Network layer need to achieve functions of data transferring, routing, transmission control, congestion control, network connecting and maintenance. It influences whether Joined Data Links can be soundly integrated into network-centric warfare.

In current tactical data links, most connections between processes are based on TDMA or polling ${ }^{[1]}$. Without TCP/IP, connections between network nodes are fixed and system is inflexible. When a new connection between 2 platforms is need, a reliable way is to add this connection at the initiation of the system, but that greatly affects the efficiency and generality of the system, difficult to solve accidental information resources access request. Therefore, adding TCP/IP into Network Layer is worth considering.

4) Link Layer

Provides point to point data transmission like TCP/IP, and establishes data links between communication entities on the base of bit transmission service provided by Physical Layer. It also uses error control and traffic control to ignore the potential physical links failure.

\section{5) Physical Layer}


It provides physical connection for Link Layer on physical medium.

6) Credibility Control Dimension

In complicated battlefield environment, when Joined Data Links based on cloud computing unilaterally and transparently provides friendly users with individualized services, how to make sure the credibility of service requester should be paid great attention to ${ }^{[22]}$. Therefore, we add Credibility Control Dimension into our architecture.

In light of trusted network ${ }^{[21]}$ concept, credible control regulates JDLCC network behavior, service, auditable protocol, source address spoofing proofing, security vulnerability analyzing and survival ability. With the Control Dimension, information transmission and behavior of service provider and customer are status monitored, result evaluable, and exception controlled. To be specific, credibility of system should include a group of attributes: from the angle of users, service security and survivability should be ensured; from the angle of design, system manageability should be secured. Comparing to traditional network security, credibility has deeper meanings: security is a claim of outer appearance; meanwhile credibility is a measurable attribute by behavior analyzing. Therefore, we believe that Credibility Control Dimension should include: user identification administration, system survival management, behavior management, system survivability management and etc.

Thus, we have proposed a Joined Data Links architecture based on cloud computing, facing multiple battlefield users, based on service, running fluently in network-centric warfare. After battlefield users of Joined Data Links submit service requests, through Cross-link Interacting Cloud Service Layer, service provider searches corresponding services according to the requests under the support of cloud-alized technology, cloud service comprehensive management technology, cloud security technology and cloud operation management technology; credibility control technology provides lifecycle technical support for the system to run securely.

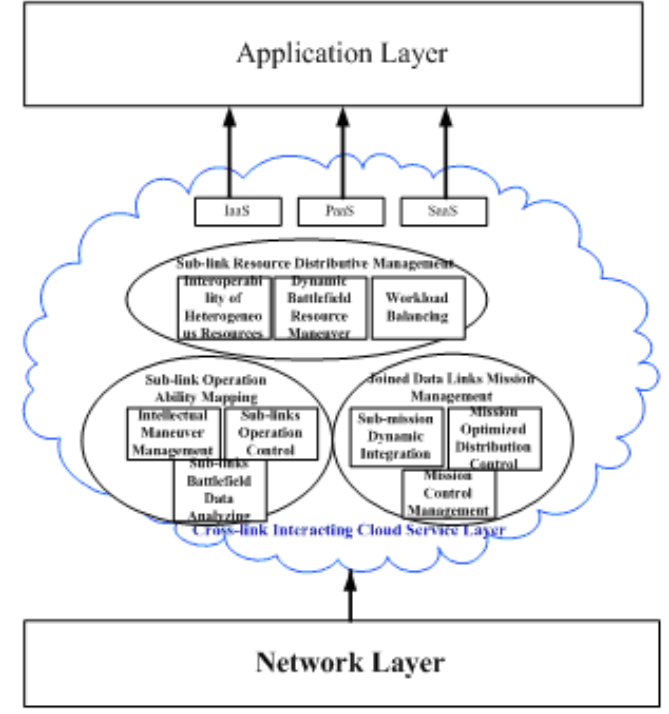

Figure 2 Cross-Link Interactive Cloud Service Layer

\section{EXPERIMENT}

We have constructed a research platform based on cloud technology, and proved that the use of cloud technology in Joined Data Links can improve the system service level markedly. But we apologize that we cannot provide the comparing results because of the secret data.

\section{CONCLUSION}

Even though Tactical data links play a very important role in modern warfare and their features are much better than those of ordinary tactical communication systems, their limitations are exist, such as specific platform oriented and lack of generality, which greatly restrict the cooperation ability between battlefield data links. Cloud computing is an innovation in information field. So we advanced a JDLCC architecture, makes tactical data links evolve into battlefield intelligent networking system. The combination converts original communication channels into "intelligent channels", achieves cooperation between multiple data links, implements "omnipresent and omnipotent" information service, satisfies the requirement of transmitting "right information" to "right receiver" in "right format" at "right time" which is emphasized by network-centric warfare, suppresses the same attempt from enemies, consequently transforms the ability of information acquisition into the ability of scientific decision making and warfare to the most extent.

\section{ACKNOWLEDGMENT}

This work was supported in part by a grant from the National Science Foundation of China under Grant No. 61173131 \& 60975015, Key Strategic Project of Chongqing of China under Grant No.CSTC2009AB2230, Strategeic Project of Chongqing of China under Grant No. 2009AC2057, The special funding for Scientific research project Of Chongqing postdoctoral researchers under Grant No. XM20120054, 2011 Specialty Research Project of Chongqing Communication College of PLA under Grant No.20110912.

\section{REFERENCES}

[1] Sun Mingyi, Yang Liping. Tactical Data Links in Information Based Warfare [M].Beijing: Post University Press, 2005.

[2] Wang Xiuhua,Wei Xin,Wang Haiyan. Network centric warfare analysis of US Army[J].Lecture Notes in Electrical Engineering, 2012, vol. 36,pp:573-578.

[3] LIU Xing, CHEN Chen, ZHOU Jin-lun, LUO Peng-cheng. An Essential-Task-Based Methodology for Development and Analysis of Information Exchange Requirements in Tactical Data Link [J]. Journal of Convergence Information Technology (JCIT), 2012, vol.7,pp:284-291.

[4] YAN Zhao-lin, HE Zhao-xiong, LUO Xu-hui, LI Hai-yan. Towards Fixed Allocation Timeslot Number Prediction Method for TDMA Tactical Data Link based on Operation Requirements.International Journal of Advancements in Computing Technology [J]. 2011, vol. 3,pp:57-64.

[5] Folks, R. L.Network Centric Warfare in the Age of Cyberspace Operations[J] .Army War Coll., Carlisle Barracks, PA. 2011, vol.5,pp:34. 
[6] Baskarada, S. Towards a Semiotic Information Position Framework for Network Centric Warfare [J]. Defence Science and Technology Organisation, Victoria (Australia).2011, vol.6,pp: 36.

[7] Rhee, Seung Hyong,Kim, Hwa-Sung; Sohn, Seung-Won.The effect of decentralized resource allocation in network-centric warfare[C].International Conference on Information Networking, 2012,pp:478-481.

[8] Casalicchio E, Galli E, Tucci S, Agent-based modelling of interdependent critical infrastructures[J]. International Journal of System of Systems Engineering, 2010, vol.2, pp :60-75.

[9] Wang, H.,Zhu, L., Mao, S., Xu, B.Research on Information Sharing Method for Future C2 in Network Centric Environment.Nanjing Research Inst. of Electronics Engineering (China). 2011, vol. 6,pp: 33.

[10] Saarelainen, Tapio, Timonen, Jussi .Tactical management in near real-time systems[C].2011 IEEE International Multi-Disciplinary Conference on Cognitive Methods in Situation Awareness and Decision Support, CogSIMA 2011,pp: 240-247.

[11] Li Fuqiang, Xu Yue. Comprehensive Application Research on Multiple Overseas Data Links [J] Modern Electronic Technology. 2010, vol. 327,pp: 96-100.

[12] Wu Zhuhua. Analyzing on Cloud Computing Core Technology [M]. Beijing: People's Post Press ,2011.

[13] Ge Junwei,Zhang Bo,Fang Yiqiu. Study on resource monitoring model in cloud computing environment[J] . Computer Engineering,2011, vol.37,pp : 31-34.

[14] Xie Haohui,Gao Ji. Research and implementation of PaaS platform based on norm-governed and policy-driven autonomic mode[J]. Application Research of Computers,2011,vol.28( 5) ,pp: 1839-1842.
[15] MELL P, GRANCE T. The NIST Definition of Cloud Computing[R].National Institute of Standards and Technology, 2011.

[16] M. Armbrust, A. Fox, R. Griffith, A. Joseph, R. Katz, A. Konwinski, G. Lee, D. Patterson, A. Rabkin, I. Stoica, M. Zaharia. Above the Clouds: A Berkeley View of Cloud computing, University of California at Berkley, USA, 2009.

[17] FOSTER I, ZHAO Y, RAICU I, et al. Cloud computing and grid computing 360-degree compared Proceedings of Grid Computing Environment s Worksho, November 12216, 2008, Austin, TX, USA: 1210.

[18] VAQUERO L M, RODERO2MERINO L, CACERES J, et al .A break in the clouds: towards a cloud definition. ACM SIGCOMM Computer Communication Review, 2009, vol.39 (1),pp :50-55.

[19] RITTINGHOUSE J W, RANSOME J F. Cloud computing: implementation, management, and security. Boca Raton, FL, USA: CRC Press, 2010.

[20] Wu Jiyi; Shen Qianli; Zhang Jianlin; Shen Zhonghua; Ping Lingdi. Cloud Computing: From Cloud Security to Credible Cloud [J] Computer Research and Development,2011, vol. S1,pp:53-61

[21] Mazda Salmanian1, Li Pan2, Jiangxin Hu2, and Ming Li. On the Efficiency of Establishing and Maintaining Security Associations in Tactical MANETs in Group Formation [C]. The 2011 Military Communications Conference .2011, pp:1176-1182.

[22] Chen Kang, Zheng Weimin. Cloud Computing: System Example and Research Status [J].Software Journal, 2009, vol.20 (5), pp: 1337-1348. 